\title{
Heavy Element Accumulation in Aquatic Beetles of the Genus Enochrus (Coleoptera: Hydrophilidae) in Erzurum Province
}

\author{
$\operatorname{Aydogan} \mathrm{Z}^{*}$, Gurol $\mathrm{A}^{2}$, and Incekara $\mathrm{U}^{3}$ \\ ${ }^{1}$ Atatürk University, Narman Vocational School, Narman, Erzurum, Turkey \\ ${ }^{2}$ Atatürk University, Department of Biology, Faculty of Science, Erzurum, Turkey \\ ${ }^{3}$ Atatürk University, Department of Physics, Faculty of Science, Erzurum, Turkey
}

*Corresponding author: Atatürk University, Narman Vocational School, 25530, Narman, Erzurum, Turkey, Tel: 05304961350,E-mail: zeybionep@hotmail.com

Citation: Aydogan Z, Gurol A, Incekara U (2018) Heavy Element Accumulation in Aquatic Beetles of the Genus Enochrus (Coleoptera: Hydrophilidae) in Erzurum Province. J Environ Pollut Control 1(1): 101. doi: 10.15744/2639-9288.1.101

Received Date: January 22, 2018 Accepted Date: September 11, 2018 Published Date: September 13, 2018

\begin{abstract}
In the present study, fourteen heavy elements ( $\mathrm{Ti}, \mathrm{V}, \mathrm{Cr}, \mathrm{Mn}, \mathrm{Fe}, \mathrm{Co}, \mathrm{Ni}, \mathrm{Cu}, \mathrm{Zn}, \mathrm{As}, \mathrm{Se}, \mathrm{Br}, \mathrm{Sr}, \mathrm{Pb}$ ) accumulation in sediment, water and Enochrus spp. species organs (total body) were evaluated. For this purpose, water, sediment and Enochrus spp. were collected from nine wetland sites in Erzurum province (Turkey). Measurements of heavy elements were made with Energy Dispersive X-Ray Fluorescence (EDXRF) spectrometer. The results showed that there are differences in element concentrations between species and stations. Insects were mostly found in industrialized areas. The water samples' heavy element levels were compared with national water quality standards. The values of some heavy elements found at higher concentration than the acceptable limits.
\end{abstract}

Keywords: Aquatic Coleoptera; EDXRF; Pollution; Wetland

\section{Introduction}

All matters (living and non-living) are made up of elements and almost every manufactured product involves the elements. Elements including heavy metals and metalloids are all occur naturally in the Earth's crust and are usually present certain degree in all environments. They are form of inorganic and organic compounds and their natural and man-made sources continuously enter the ecosystem and pose a serious damage because of their long time persistence, toxicity and tendency to bioaccumulation, bioconcentration and biomagnification in the food chain [1]. This element accumulation in the food chain creates increasing pressure on all living things every passing day. In the future, it seems that the situation will become more dramatic. Like other ecosystems pollutions, aquatic ecosystems pollution with these contaminants has become a matter of great concern over the last decades. Accumulation occurs in all aquatic invertebrates' tissues, whether or not these elements are essential to metabolism and if the certain threshold concentrations are exceeded, it becomes toxic $[2,3]$.

The terms bioindicator and biological monitor have significant variation in meaning. Bioindicator generally refers to organisms or molecules that provide significant information in regard to quality of environment or environmental changes, whereas biomonitors are the organisms that provide quantitative information about the environment. In this regard, a biomonitor always acts as a bioindicator but a bioindicator does not always a biomonitor [4]. According to Witting, Markert and Rainbow [2,5,6], there are some basic criteria for a suitable biomonitor that it should; have large numbers all over the studying area, have cosmopolitan geographical distribution, be easy and inexpensive to sampling and have well known taxonomy. Today to evaluate ecosystem condition and its quality, biological monitoring methods have been applied widely in ecological research [6-8].

Like other organisms in the ecosystems, insects are exposed to various anthropogenic chemicals and some of them accumulate these chemicals in their body. Insects are largest group of organisms, and have numerous effects on human health because of diversity in their morphology and physiology, their abundance, economic importance [9]. Aquatic insects live or spend a part of their life cycle in different kind of water bodies [10]. Aquatic insects are good indicators of water qualities since they have various environmental tolerant levels [11]. Some of them such as midge and blackfly larva etc. indicate poor water quality and can live in moderately or extremely polluted waters, whereas some of them such as mayfly, stonefly nymph very vulnerable and sensitive to pollution and indicates good water quality [12-14] investigated metal contamination ability of the Odonata larvae and the study suggests that they have an ability to take up metals and appropriate indicator for ecosystem health. Boyd (2009) studied Ni 
hyper-accumulator insects which feed on Ni hyper-accumulator plants showed that Ni hyper-accumulator insects accumulate more Ni than other insects. Aydoğan et al. (2017) [8] studied heavy metal accumulation in some aquatic insects and tissues of Chondrostoma regium in the same habitat showed that insects accumulated more metals than their environment and due to food of studied fish the metals could be transfer from insect to along the food web.

Hydrophilidae is one of the largest families of Coleoptera, and some feature of Hydrophilidae make them good biomonitor for heavy element such as it has worldwide distribution, well known taxonomy and larvae and adults live in different environmental conditions. Hydrophilidae are water scavenger beetles and generally occur in shallower regions of wetland with abundant macrophytes and feed mainly on detritus, algae and decaying organic matter in contrast to adults, larvae are predaceous, preying on various invertebrates [15]. Hydrophilidae are present in low dissolved oxygen content water indicating the hardy nature of these beetles [16]. The aquatic Coleoptera of the genus Enochrus Thomson 1859 are among the commonest species of the European hydrophilids, and also is the third largest genus in the Hydrophilinae with 223 species placed in six subgenera worldwide [17]. This research attempted to evaluate the potential use of adults Enochrus spp. samples as biomonitor of heavy element pollution, as well as classify and compare the health status of freshwater in Erzurum province, Turkey.

\section{Materials and Methods}

Erzurum is the biggest city of the Eastern Anatolia Region in Turkey. In addition, it is one of the highest and coldest cities in Turkey. It is very rich in terms of water resources. Industrialization has not improved but economy mostly based on agricultural and livestock activities, trade and winter tourism. In this study, nine different locations (i.e; industrial, roadside, urban, suburban and rural area) were selected. These localities are described in Table 1 and given in Figure 1.

\begin{tabular}{|c|c|c|c|c|}
\hline $\begin{array}{l}\text { Station } \\
\text { Number }\end{array}$ & $\begin{array}{l}\text { Altitude } \\
\text { (m) }\end{array}$ & Coordinates & Station information & $\begin{array}{l}\text { Notes on near } \\
\text { environment }\end{array}$ \\
\hline 1 & 1636 & $\begin{array}{l}39^{\circ} 54^{\prime} 19^{\prime \prime} \mathrm{N} \\
40^{\circ} 41^{\prime} 01^{\prime \prime} \mathrm{E}\end{array}$ & $\begin{array}{l}500 \mathrm{~m} \text { Western front of } \\
\text { Cement factory }\end{array}$ & $\begin{array}{l}\text { Traffic and ash emission } \\
\text { from cement factory }\end{array}$ \\
\hline 2 & 1669 & $\begin{array}{l}39^{\circ} 54^{\prime} 38^{\prime \prime} \mathrm{N} \\
40^{\circ} 41^{\prime} 41^{\prime \prime} \mathrm{E}\end{array}$ & $\begin{array}{l}500 \mathrm{~m} \text { Eastern front of } \\
\text { Cement factory }\end{array}$ & $\begin{array}{l}\text { Ash emission from } \\
\text { cement factory }\end{array}$ \\
\hline 3 & 1636 & $\begin{array}{l}39^{\circ} 56^{\prime} 41^{\prime \prime} \mathrm{N} \\
40^{\circ} 39^{\prime} 44^{\prime \prime} \mathrm{E}\end{array}$ & $\begin{array}{c}\text { Aşkale-Trabzon } \\
\text { Highway }\end{array}$ & $\begin{array}{l}\text { Traffic pollution, lim- } \\
\text { ited human activities }\end{array}$ \\
\hline 4 & 1759 & $\begin{array}{l}39^{\circ} 56^{\prime} 03^{\prime \prime} \mathrm{N} \\
41^{\circ} 04^{\prime} 24^{\prime \prime} \mathrm{E}\end{array}$ & $\begin{array}{c}\text { Sugar factory Drainage } \\
\text { channel }\end{array}$ & $\begin{array}{l}\text { Sugar factory } \\
\text { waste }\end{array}$ \\
\hline 5 & 1754 & $\begin{array}{l}39^{\circ} 57^{\prime} 04^{\prime \prime} \mathrm{N} \\
41^{\circ} 04^{\prime} 39^{\prime \prime} \mathrm{E}\end{array}$ & Erzurum-Ilıca Highway & $\begin{array}{l}\text { Sugar factory waste and } \\
\text { traffic pollution }\end{array}$ \\
\hline 6 & 1765 & $\begin{array}{l}40^{\circ} 02^{\prime} 31^{\prime \prime} \mathrm{N} \\
41^{\circ} 21^{\prime} 05^{\prime \prime} \mathrm{E}\end{array}$ & $\begin{array}{c}\text { Erzurum-Tortum } \\
\text { Highway }\end{array}$ & $\begin{array}{l}\text { Traffic and domestic } \\
\text { pollution }\end{array}$ \\
\hline 7 & 1819 & $\begin{array}{l}40^{\circ} 04^{\prime} 35^{\prime \prime} \mathrm{N} \\
41^{\circ} 21^{\prime} 38^{\prime \prime} \mathrm{E} \\
\end{array}$ & Gökçeyamaç Village & $\begin{array}{l}\text { Agriculture, livestock } \\
\text { and domestic pollution }\end{array}$ \\
\hline 8 & 1826 & $\begin{array}{l}40^{\circ} 06^{\prime} 05^{\prime \prime} \mathrm{N} \\
41^{\circ} 21^{\prime} 36^{\prime \prime} \mathrm{E}\end{array}$ & $\begin{array}{l}\text { Akdağ Village (Erzu- } \\
\text { rum) }\end{array}$ & $\begin{array}{l}\text { Contamination due } \\
\text { to livestock }\end{array}$ \\
\hline 9 & 1766 & $\begin{array}{l}39^{\circ} 55^{\prime} 59^{\prime \prime} \mathrm{N} \\
41^{\circ} 08^{\prime} 56^{\prime \prime} \mathrm{E}\end{array}$ & Dadaşkent (Erzurum) & Heavy traffic pollution \\
\hline
\end{tabular}

Table 1: Description of the nine sampling sites in Erzurum province

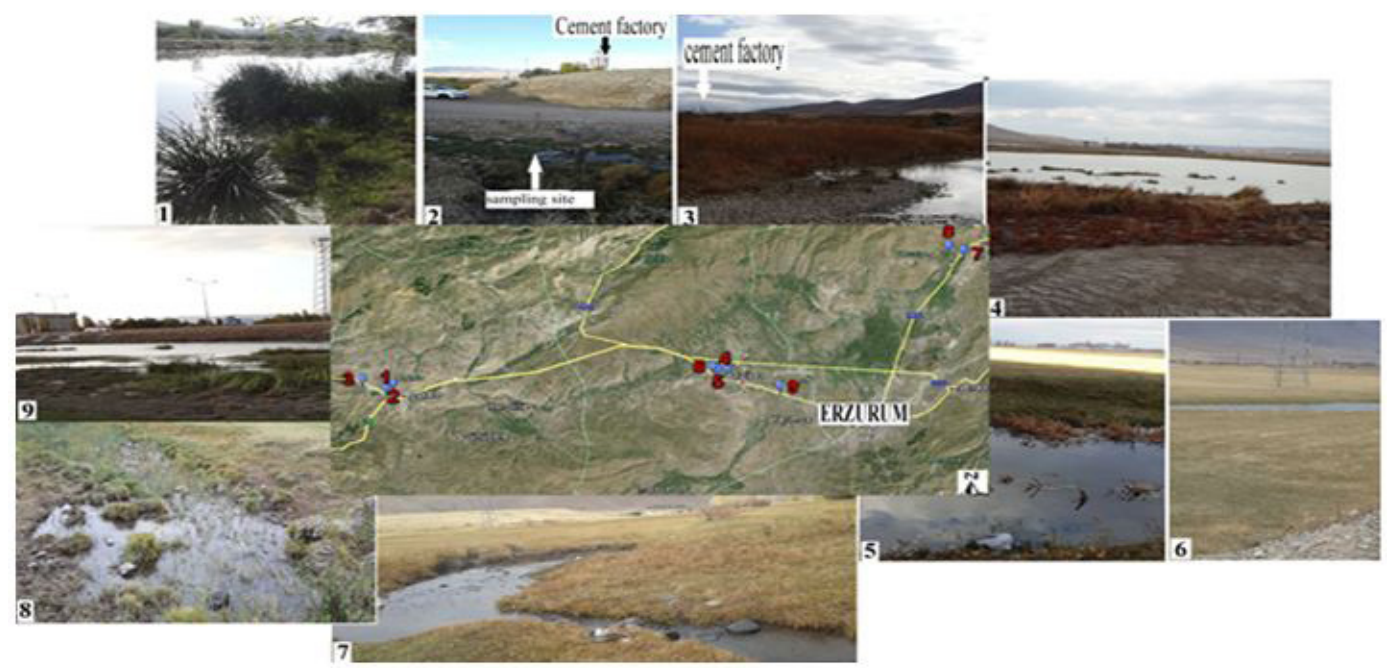

Figure 1: Photos of the nine sampling sites and location in Erzurum province 
To determine the extent of element accumulation in nine sites of Erzurum, all samples (Enochrus spp. and their abiotic environment) were collected in June, July and August 2014. The samples were collected, identified and analyzed as described in Aydoğan et al. $(2016 ; 2017[8,18]) .1 \mathrm{~mm}$ mesh aperture sieve and mouth aspirator was used to collect insect samples and they were preserved in $70 \%$ alcohol. Identification was made under stereomicroscope by using male genitalia of insects. The aquatic insects were identified with using taxonomical key to the species level $[19,20]$. The sediments were taken from $30 \mathrm{~cm}$ depth benthic zone. The water samples were taken from $0.5 \mathrm{~m}$ depth of stream. $15 \mathrm{cc}$ for water and $3 \mathrm{gr}$ for sediment was enough to analyze in EDXRF. Water and sediment samples were kept in the refrigerator until analysis.

After identification, samples were dried in an oven at $80{ }^{\circ} \mathrm{C}$ during $36 \mathrm{~h}$. Concentrations of 14 elements in the samples measured by EDXRF spectrometry. To determine heavy elements, $1 \mathrm{Ci}^{241} \mathrm{Am}$ radioactive source and an $\mathrm{HPGe}$ detector with resolution $\sim 180$ $\mathrm{eV}$ at $59.5 \mathrm{keV}$ was used. All samples were excited by using $59.5 \mathrm{keV}$ photons, emitted from ${ }^{241}$ Am radioactive source. All measurements were carried out under vacuum. Water and sediments samples were put in Al sample holder with Mylar films on both sides. Measurement time for water and sediment samples was $4 \mathrm{~h}$. Insects were pulverized, and then, cellulose was added as a binder. Five tons of pressure was applied to make $13 \mathrm{~mm}$ diameter pellets of each species. WinAXIL program is used for determinate the concentration of elements in each sample. The model parameters are optimized by means of a nonlinear least squares strategy, using a modified Marquardt algorithm to minimize the weighted (optional choice) sum of differences $\chi^{2}$ between the experimental data and the mathematical model. The concentration presented in this table has some uncertainties due to EDXRF (maximum $~ 5 \%$ ). Possible error sources for these uncertainties are given in Table 2, and the typical spectrum of samples in EDXRF shown in Figure 2. Chemical parameters of the insects, water and sediment samples are given in Table 3.

\begin{tabular}{|c|c|}
\hline Nature of Uncertainty & $\begin{array}{c}\text { Uncertainty } \\
(\%)\end{array}$ \\
\hline Counting Statistics & $\sim 1.00$ \\
\hline Systematic errors & $\sim 2.00$ \\
\hline $\begin{array}{c}\text { Peak evaluation } \\
\text { procedure }\end{array}$ & $\sim 3.00$ \\
\hline $\begin{array}{c}\text { Fundamental parameter } \\
\text { methods }\end{array}$ & $\sim 3.00$ \\
\hline
\end{tabular}

Table 2: Error sources in the experimental results

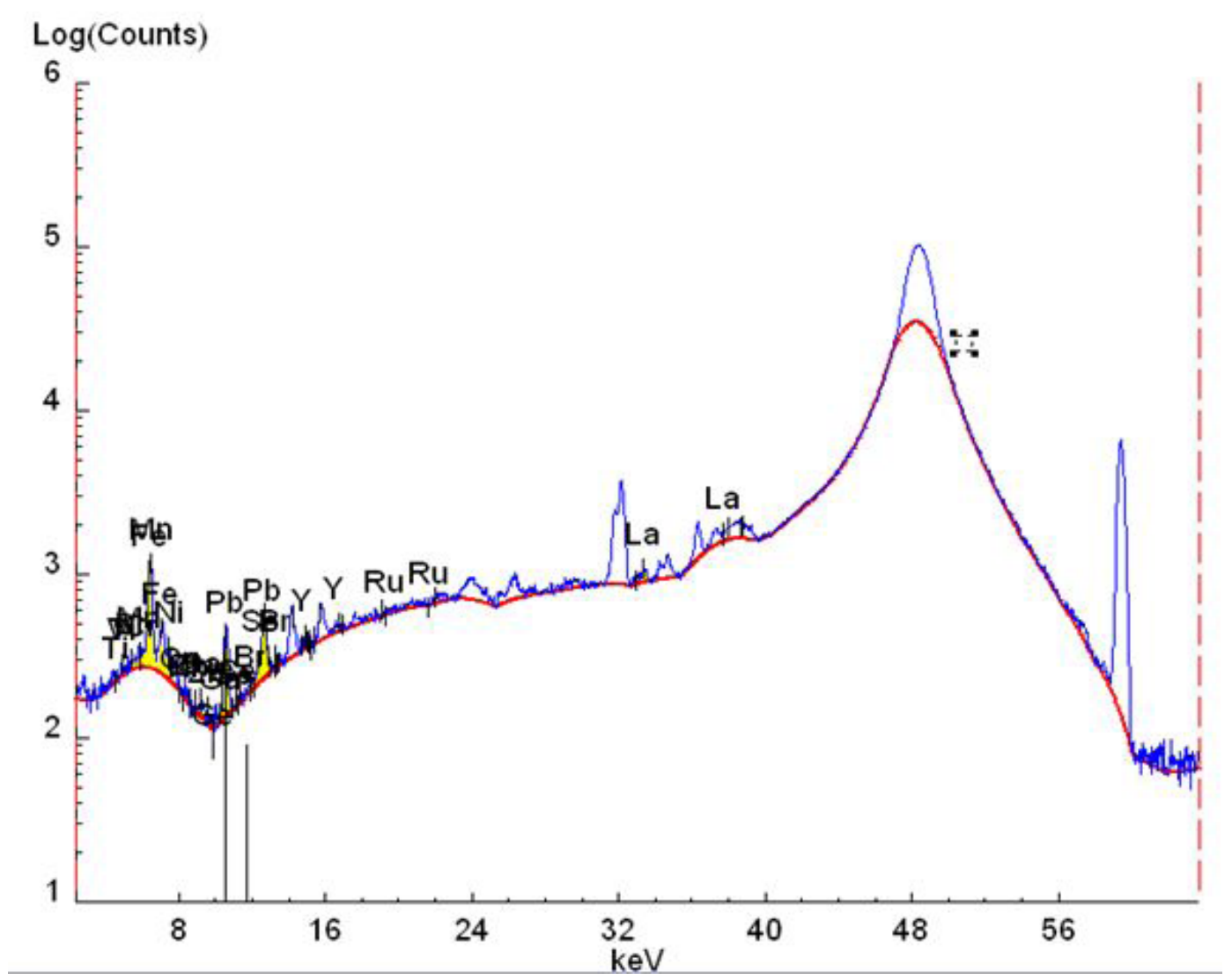

Figure 2: The typical spectrum of a sample in EDXRF 


\begin{tabular}{|c|c|c|c|c|c|c|c|c|c|c|c|c|c|c|c|}
\hline \multirow{2}{*}{$\begin{array}{l}\text { Sta- } \\
\text { tions }\end{array}$} & \multirow[t]{2}{*}{ Samples } & \multicolumn{14}{|c|}{ Mean \pm SD } \\
\hline & & $\mathrm{Ti}$ & V & $\mathrm{Cr}$ & Mn & $\mathrm{Fe}$ & Co & $\mathrm{Ni}$ & $\mathrm{Cu}$ & Zn & As & Se & $\mathrm{Br}$ & $\mathrm{Sr}$ & $\mathbf{P b}$ \\
\hline \multirow[t]{4}{*}{1} & Water & $\begin{array}{c}436 \pm \\
6.55\end{array}$ & $\begin{array}{l}82.6 \\
\pm 4.5\end{array}$ & $\begin{array}{c}22 \\
\pm 2.6\end{array}$ & $\begin{array}{l}6.2 \pm \\
0.26\end{array}$ & $\begin{array}{l}2.9 \pm \\
0.15\end{array}$ & $\begin{array}{l}1 \pm \\
0.1\end{array}$ & $\begin{array}{c}0.3 \\
\pm 0.2 \\
\end{array}$ & $\begin{array}{c}0.36 \\
\pm 0.15 \\
\end{array}$ & $0 \pm 0$ & $\begin{array}{c}0.2 \\
\pm 0.1 \\
\end{array}$ & $0 \pm 0$ & $\begin{array}{l}0.34 \\
\pm 0.1\end{array}$ & $0 \pm 0$ & $\begin{array}{l}1.7 \pm \\
0.25\end{array}$ \\
\hline & Sediment & $\begin{array}{l}1.7 \pm \\
0.25\end{array}$ & $\begin{array}{c}0.32 \\
\pm 0.11 \\
\end{array}$ & $\begin{array}{c}849 \\
\pm 6.02 \\
\end{array}$ & $0 \pm 0$ & $\begin{array}{c}0.13 \pm \\
0.06\end{array}$ & $0 \pm 0$ & $\begin{array}{r}22.3 \\
\pm 2.5 \\
\end{array}$ & $\begin{array}{c}9.23 \pm \\
0.25\end{array}$ & $\begin{array}{l}4.9 \pm \\
0.65\end{array}$ & $\begin{array}{c}9.5 \\
\pm 0.4 \\
\end{array}$ & $\begin{array}{c}10.06 \\
\pm 0.5\end{array}$ & $\begin{array}{l}5.03 \\
\pm 0.4 \\
\end{array}$ & $\begin{array}{l}9.6 \pm \\
0.36\end{array}$ & $\begin{array}{r}10.8 \pm \\
1.04 \\
\end{array}$ \\
\hline & $\begin{array}{l}\text { E. quadri- } \\
\text { punctatus }\end{array}$ & $\begin{array}{c}127 \pm \\
5.5\end{array}$ & $\begin{array}{c}23.3 \\
\pm 3.05 \\
\end{array}$ & $\begin{array}{l}18.1 \\
\pm 2.8\end{array}$ & $\begin{array}{c}6.8 \pm \\
1.2 \\
\end{array}$ & $\begin{array}{l}2.8 \pm \\
0.41\end{array}$ & $\begin{array}{c}2.33 \pm \\
0.41\end{array}$ & $\begin{array}{l}0.41 \\
\pm 0.2 \\
\end{array}$ & $\begin{array}{c}0.42 \\
\pm 0.06 \\
\end{array}$ & $\begin{array}{c}0.2 \pm \\
0.1\end{array}$ & $0 \pm 0$ & $\begin{array}{l}0.24 \\
\pm 0.1\end{array}$ & $0 \pm 0$ & $\begin{array}{c}320.6 \\
\pm 6.1\end{array}$ & $\begin{array}{c}602 \pm \\
7.21\end{array}$ \\
\hline & $\begin{array}{l}\text { E. halophi- } \\
\text { lus }\end{array}$ & $\begin{array}{c}307 \pm \\
8.18\end{array}$ & $\begin{array}{l}55.3 \\
\pm 3.5 \\
\end{array}$ & $\begin{array}{l}46.6 \\
\pm 3.5 \\
\end{array}$ & $19 \pm 2$ & $\begin{array}{c}1.2 \\
\pm 0.2 \\
\end{array}$ & $\begin{array}{c}5.2 \pm \\
0.2 \\
\end{array}$ & $\begin{array}{c}5.1 \\
\pm 0.4 \\
\end{array}$ & $\begin{array}{c}1.03 \\
\pm 0.14 \\
\end{array}$ & $\begin{array}{l}0.47 \\
\pm 0.3\end{array}$ & $0 \pm 0$ & $\begin{array}{c}1.2 \\
\pm 0.2\end{array}$ & $\begin{array}{c}0.6 \\
\pm 0.3 \\
\end{array}$ & $\begin{array}{c}0.14 \\
\pm 0.05\end{array}$ & $\begin{array}{l}0.2 \pm \\
0.01\end{array}$ \\
\hline \multirow[t]{8}{*}{2} & Water & $\begin{array}{c}321.8 \pm \\
20\end{array}$ & $\begin{array}{l}63.8 \\
\pm 2.7\end{array}$ & $\begin{array}{l}14.6 \\
\pm 2.5\end{array}$ & $\begin{array}{c}4.9 \pm \\
0.6\end{array}$ & $\begin{array}{c}2.2 \\
\pm 0.2\end{array}$ & $\begin{array}{c}0.03 \pm \\
0.02\end{array}$ & $\begin{array}{c}0.5 \\
\pm 0.1 \\
\end{array}$ & $\begin{array}{c}0.13 \\
\pm 0.05\end{array}$ & $\begin{array}{c}0.2 \pm \\
0.1 \\
\end{array}$ & $\begin{array}{c}0.2 \\
\pm 0.1 \\
\end{array}$ & $0 \pm 0$ & $\begin{array}{l}0.25 \\
\pm 0.1\end{array}$ & $0 \pm 0$ & $\begin{array}{c}1.9 \\
\pm 0.2\end{array}$ \\
\hline & Sediment & $\begin{array}{l}1.2 \pm \\
0.25 \\
\end{array}$ & $0 \pm 0$ & $\begin{array}{c}663.6 \\
\pm 13 \\
\end{array}$ & $\begin{array}{c}231.3 \\
\pm 12 \\
\end{array}$ & $\begin{array}{r}244 \\
\pm 4.5 \\
\end{array}$ & $\begin{array}{c}8.8 \pm \\
0.4\end{array}$ & $\begin{array}{l}15 \\
\pm 3 \\
\end{array}$ & $\begin{array}{c}7.5 \pm \\
0.4 \\
\end{array}$ & $\begin{array}{c}3.7 \pm \\
0.3 \\
\end{array}$ & $\begin{array}{r}7.3 \\
\pm 0.7 \\
\end{array}$ & $\begin{array}{c}8.2 \\
\pm 0.3 \\
\end{array}$ & $\begin{array}{c}3.6 \\
\pm 0.5 \\
\end{array}$ & $\begin{array}{c}9.5 \\
\pm 0.5 \\
\end{array}$ & $\begin{array}{c}2.5 \\
\pm 0.5 \\
\end{array}$ \\
\hline & E. ater & $\begin{array}{l}1.1 \pm \\
0.16\end{array}$ & $0 \pm 0$ & $\begin{array}{r}434 \\
\pm 6.1 \\
\end{array}$ & $0 \pm 0$ & $\begin{array}{c}185.3 \\
\pm 4.1 \\
\end{array}$ & $0 \pm 0$ & $\begin{array}{c}9.4 \\
\pm 0.3 \\
\end{array}$ & $0 \pm 0$ & $\begin{array}{c}2.2 \pm \\
0.2 \\
\end{array}$ & $\begin{array}{l}4 \pm \\
0.4 \\
\end{array}$ & $\begin{array}{c}4.4 \\
\pm 0.7 \\
\end{array}$ & $\begin{array}{l}10.1 \\
\pm 0.8 \\
\end{array}$ & $\begin{array}{c}0.5 \\
\pm 0.3 \\
\end{array}$ & $\begin{array}{r}175.6 \\
\pm 5.5 \\
\end{array}$ \\
\hline & E. bicolor & $\begin{array}{l}0.1 \pm \\
0.02\end{array}$ & $0 \pm 0$ & $\begin{array}{l}73.6 \\
\pm 3.5 \\
\end{array}$ & $0 \pm 0$ & $\begin{array}{l}87.6 \\
\pm 2.5 \\
\end{array}$ & $0 \pm 0$ & $\begin{array}{c}1.5 \\
\pm 0.3 \\
\end{array}$ & $\begin{array}{c}0.6 \\
\pm 0.2 \\
\end{array}$ & $\begin{array}{c}0.5 \pm \\
0.3\end{array}$ & $\begin{array}{c}1.5 \\
\pm 0.1\end{array}$ & $\begin{array}{c}0.5 \\
\pm 0.4 \\
\end{array}$ & $\begin{array}{c}1.9 \pm \\
0.2\end{array}$ & $\begin{array}{c}0.2 \pm \\
0.1\end{array}$ & $\begin{array}{c}10.4 \\
\pm 1.05 \\
\end{array}$ \\
\hline & $\begin{array}{l}\text { E. halophi- } \\
\text { lus }\end{array}$ & $\begin{array}{c}147 \pm \\
4.53 \\
\end{array}$ & $0 \pm 0$ & $0 \pm 0$ & $0 \pm 0$ & $\begin{array}{c}3.1 \\
\pm 0.4 \\
\end{array}$ & $0 \pm 0$ & $\begin{array}{c}0.13 \\
\pm 0.05 \\
\end{array}$ & $\begin{array}{c}0.2 \\
\pm 0.17 \\
\end{array}$ & $0 \pm 0$ & $\begin{array}{c}0.1 \\
\pm 0.5 \\
\end{array}$ & $\begin{array}{l}0.13 \\
0.05 \\
\end{array}$ & $\begin{array}{c}0.14 \\
\pm 0.05 \\
\end{array}$ & $\begin{array}{c}0.06 \pm \\
0.02 \\
\end{array}$ & $\begin{array}{c}1.8 \\
\pm 0.4 \\
\end{array}$ \\
\hline & $\begin{array}{l}\text { E. segmen- } \\
\text { tinotatus }\end{array}$ & $\begin{array}{c}173 \pm \\
3.8\end{array}$ & $0 \pm 0$ & $\begin{array}{c}7.2 \\
\pm 0.2 \\
\end{array}$ & $\begin{array}{c}2.2 \\
\pm 0.2 \\
\end{array}$ & $\begin{array}{c}3.1 \\
\pm 0.2 \\
\end{array}$ & $0 \pm 0$ & $\begin{array}{c}0.13 \\
\pm 0.05\end{array}$ & $\begin{array}{c}0.2 \\
\pm 0.1\end{array}$ & $0 \pm 0$ & $\begin{array}{c}0.2 \\
\pm 0.1 \\
\end{array}$ & $\begin{array}{c}0.1 \\
\pm 0.05 \\
\end{array}$ & $\begin{array}{c}0.1 \\
\pm 0.05 \\
\end{array}$ & $\begin{array}{c}0.01 \\
\pm 0.003 \\
\end{array}$ & $\begin{array}{c}2.3 \\
\pm 0.2 \\
\end{array}$ \\
\hline & $\begin{array}{l}\text { E. fusci- } \\
\text { pennis }\end{array}$ & $\begin{array}{l}0.2 \pm \\
0.06 \\
\end{array}$ & $0 \pm 0$ & $0 \pm 0$ & $0 \pm 0$ & $\begin{array}{c}190 \\
\pm 10.4 \\
\end{array}$ & $0 \pm 0$ & $\begin{array}{c}2.2 \\
\pm 0.2 \\
\end{array}$ & $\begin{array}{c}1.4 \\
\pm 0.2 \\
\end{array}$ & $\begin{array}{c}4.2 \pm \\
0.2 \\
\end{array}$ & $\begin{array}{c}1.6 \\
\pm 0.1 \\
\end{array}$ & $\begin{array}{l}1.03 \\
\pm 0.1 \\
\end{array}$ & $\begin{array}{c}4.1 \\
\pm 0.3 \\
\end{array}$ & $\begin{array}{c}1.1 \\
\pm 0.1 \\
\end{array}$ & $\begin{array}{l}11.4 \\
\pm 0.9 \\
\end{array}$ \\
\hline & $\begin{array}{l}\text { E. quadri- } \\
\text { punctatus }\end{array}$ & $\begin{array}{c}783 \pm \\
4.3 \\
\end{array}$ & $0 \pm 0$ & $\begin{array}{c}32 \\
\pm 2.08 \\
\end{array}$ & $\begin{array}{l}10.1 \\
\pm 0.8 \\
\end{array}$ & $\begin{array}{r}30.6 \\
\pm 3.5 \\
\end{array}$ & $0 \pm 0$ & $\begin{array}{c}0.4 \\
\pm 0.3 \\
\end{array}$ & $\begin{array}{c}0.2 \\
\pm 0.1 \\
\end{array}$ & $\begin{array}{l}1.1 \pm \\
0.17\end{array}$ & $\begin{array}{c}0.2 \\
\pm 0.1 \\
\end{array}$ & $\begin{array}{c}0.26 \pm \\
0.15\end{array}$ & $\begin{array}{c}0.5 \\
\pm 0.2 \\
\end{array}$ & $\begin{array}{l}0.1 \pm \\
0.05 \\
\end{array}$ & $\begin{array}{c}6.2 \\
\pm 0.2 \\
\end{array}$ \\
\hline \multirow[t]{5}{*}{3} & Water & $\begin{array}{c}796.8 \\
\pm 5 \\
\end{array}$ & $\begin{array}{c}140.5 \\
\pm 8 \\
\end{array}$ & $\begin{array}{l}10.3 \\
\pm 0.2 \\
\end{array}$ & $\begin{array}{l}10.1 \\
\pm 0.7 \\
\end{array}$ & $\begin{array}{c}4.8 \\
\pm 0.4 \\
\end{array}$ & $\begin{array}{c}1.8 \pm \\
0.2 \\
\end{array}$ & $\begin{array}{c}0.7 \\
\pm 0.2 \\
\end{array}$ & $\begin{array}{c}0.2 \\
\pm 0.1 \\
\end{array}$ & $\begin{array}{c}0.2 \pm \\
0.1 \\
\end{array}$ & $\begin{array}{c}0.2 \\
\pm 0.1 \\
\end{array}$ & $0 \pm 0$ & $\begin{array}{c}0.2 \\
\pm 0.05 \\
\end{array}$ & $0 \pm 0$ & $\begin{array}{r}3.23 \\
\pm 0.2 \\
\end{array}$ \\
\hline & Sediment & $\begin{array}{c}1.2 \pm \\
0.2\end{array}$ & $0 \pm 0$ & $\begin{array}{c}662 \\
\pm 14.8 \\
\end{array}$ & $\begin{array}{c}240.3 \\
\pm 3\end{array}$ & $\begin{array}{c}245 \\
\pm 4.5 \\
\end{array}$ & $\begin{array}{c}9.9 \pm \\
1.1\end{array}$ & $\begin{array}{l}16.6 \\
\pm 1.5 \\
\end{array}$ & $\begin{array}{c}7.8 \\
\pm 0.2 \\
\end{array}$ & $\begin{array}{c}3.9 \pm \\
0.6\end{array}$ & $\begin{array}{c}6.5 \pm \\
1.3 \\
\end{array}$ & $\begin{array}{c}7.5 \pm \\
0.4 \\
\end{array}$ & $\begin{array}{c}4.3 \\
\pm 0.3\end{array}$ & $\begin{array}{c}10 \pm \\
0.8\end{array}$ & $\begin{array}{c}2.4 \\
\pm 0.5\end{array}$ \\
\hline & $\begin{array}{l}\text { E. halophi- } \\
\text { lus }\end{array}$ & $\begin{array}{c}3.6 \pm \\
0.4 \\
\end{array}$ & $0 \pm 0$ & $\begin{array}{c}0.1 \\
\pm 0.02 \\
\end{array}$ & $\begin{array}{l}494.5 \\
\pm 13 \\
\end{array}$ & $\begin{array}{c}557.3 \\
\pm 11 \\
\end{array}$ & $\begin{array}{r}72 \pm \\
2.2 \\
\end{array}$ & $\begin{array}{r}30.3 \\
\pm 1.5 \\
\end{array}$ & $0 \pm 0$ & $\begin{array}{c}8.6 \pm \\
0.5 \\
\end{array}$ & $\begin{array}{l}11 \pm \\
1.8 \\
\end{array}$ & $\begin{array}{l}15.6 \\
\pm 0.7 \\
\end{array}$ & $16 \pm 0.7$ & $\begin{array}{c}2.2 \pm \\
0.3 \\
\end{array}$ & $\begin{array}{r}128.6 \\
\pm 3.2 \\
\end{array}$ \\
\hline & $\begin{array}{l}\text { E. fusci- } \\
\text { pennis }\end{array}$ & $\begin{array}{l}0.1 \pm \\
0.02 \\
\end{array}$ & $0 \pm 0$ & $\begin{array}{l}42.6 \\
\pm 2.5 \\
\end{array}$ & $\begin{array}{l}12.3 \\
\pm 2.5 \\
\end{array}$ & $20 \pm 2$ & $0 \pm 0$ & $\begin{array}{c}0.5 \\
\pm 0.4 \\
\end{array}$ & $\begin{array}{l}0.33 \\
\pm 0.2 \\
\end{array}$ & $\begin{array}{l}0.2 \pm \\
0.07\end{array}$ & $\begin{array}{c}0.2 \\
\pm 0.1 \\
\end{array}$ & $\begin{array}{c}0.3 \\
\pm 0.2 \\
\end{array}$ & $\begin{array}{c}0.5 \\
\pm 0.06 \\
\end{array}$ & $\begin{array}{c}0.13 \pm \\
0.05\end{array}$ & $\begin{array}{c}4.4 \\
\pm 0.1 \\
\end{array}$ \\
\hline & E. bicolor & $0 \pm 0$ & $\begin{array}{c}0.5 \\
\pm 0.1 \\
\end{array}$ & $\begin{array}{c}0.1 \\
\pm 0.02 \\
\end{array}$ & $\begin{array}{c}463.6 \\
\pm 13 \\
\end{array}$ & $\begin{array}{c}755.6 \\
\pm 4 \\
\end{array}$ & $0 \pm 0$ & $\begin{array}{l}29.8 \\
\pm 0.7\end{array}$ & $\begin{array}{l}15.3 \\
\pm 0.2 \\
\end{array}$ & $\begin{array}{c}8.3 \\
\pm 0.3 \\
\end{array}$ & $\begin{array}{c}13 \\
\pm 0.7 \\
\end{array}$ & $\begin{array}{c}15 \pm \\
0.5 \\
\end{array}$ & $\begin{array}{l}28.3 \\
\pm 1.5 \\
\end{array}$ & $\begin{array}{c}3.2 \\
\pm 0.2 \\
\end{array}$ & $\begin{array}{r}108.6 \\
\pm 3.2 \\
\end{array}$ \\
\hline \multirow[t]{5}{*}{4} & Water & $\begin{array}{c}239 \pm \\
3.6\end{array}$ & $45 \pm 3$ & $\begin{array}{c}10.2 \pm \\
0.6\end{array}$ & $\begin{array}{c}3.3 \\
\pm 0.2\end{array}$ & $\begin{array}{c}2.1 \\
\pm 0.4\end{array}$ & $\begin{array}{c}0.3 \\
\pm 0.1 \\
\end{array}$ & $\begin{array}{c}0.2 \\
\pm 0.1\end{array}$ & $\begin{array}{l}0.1 \pm \\
0.05\end{array}$ & $\begin{array}{l}0.1 \\
\pm 0\end{array}$ & $\begin{array}{c}0.1 \\
\pm 0.5 \\
\end{array}$ & $\begin{array}{c}0.1 \\
\pm 0.1\end{array}$ & $\begin{array}{l}0.1 \pm \\
0.04\end{array}$ & $0 \pm 0$ & $\begin{array}{l}0.9 \pm \\
0.05\end{array}$ \\
\hline & Sediment & $1 \pm 0.1$ & $0 \pm 0$ & $\begin{array}{c}467 \\
\pm 18.5 \\
\end{array}$ & $0 \pm 0$ & $\begin{array}{c}0.1 \\
\pm 0.01 \\
\end{array}$ & $0 \pm 0$ & $\begin{array}{c}11 \\
\pm 1.05 \\
\end{array}$ & $0 \pm 0$ & $\begin{array}{c}3.1 \\
\pm 0.1 \\
\end{array}$ & $\begin{array}{c}6.2 \\
\pm 0.2 \\
\end{array}$ & $\begin{array}{c}6.4 \pm \\
0.4\end{array}$ & $\begin{array}{c}3.3 \pm \\
0.2 \\
\end{array}$ & $\begin{array}{l}12.5 \\
\pm 0.5 \\
\end{array}$ & $\begin{array}{c}2.03 \pm \\
0.05\end{array}$ \\
\hline & $\begin{array}{l}\text { E. quadri- } \\
\text { punctatus }\end{array}$ & $\begin{array}{c}13.9 \pm \\
1.6\end{array}$ & $\begin{array}{c}3.2 \\
\pm 0.2 \\
\end{array}$ & $\begin{array}{c}0.5 \pm \\
0.1\end{array}$ & $\begin{array}{l}0.1 \pm \\
0.05\end{array}$ & $\begin{array}{c}0.1 \\
\pm 0.01 \\
\end{array}$ & $0 \pm 0$ & $\begin{array}{l}125 \\
\pm 3 \\
\end{array}$ & $\begin{array}{c}60.6 \\
\pm 4.04 \\
\end{array}$ & $\begin{array}{l}45 \pm \\
4.04 \\
\end{array}$ & $\begin{array}{c}56 \\
\pm 1.7 \\
\end{array}$ & $\begin{array}{c}67.5 \pm \\
2.2 \\
\end{array}$ & $\begin{array}{c}167 \\
\pm 3.6 \\
\end{array}$ & $\begin{array}{c}5.7 \\
\pm 0.2 \\
\end{array}$ & $\begin{array}{r}250.3 \\
\pm 1.5 \\
\end{array}$ \\
\hline & E. bicolor & $\begin{array}{c}390 \pm \\
2.5 \\
\end{array}$ & $72 \pm 2$ & $\begin{array}{l}15.6 \\
\pm 0.7 \\
\end{array}$ & $\begin{array}{c}4.9 \\
\pm 0.2 \\
\end{array}$ & $\begin{array}{l}3 \pm \\
0.3 \\
\end{array}$ & $\begin{array}{c}0.5 \pm \\
0.1 \\
\end{array}$ & $\begin{array}{c}0.2 \\
\pm 0.1 \\
\end{array}$ & $\begin{array}{c}0.1 \\
\pm 0.05 \\
\end{array}$ & $\begin{array}{c}0.6 \pm \\
0.2 \\
\end{array}$ & $\begin{array}{c}0.2 \pm \\
0.1 \\
\end{array}$ & $\begin{array}{l}0.1 \pm \\
0.05 \\
\end{array}$ & $\begin{array}{c}0.5 \\
\pm 0.1 \\
\end{array}$ & $0 \pm 0$ & $\begin{array}{c}3.2 \\
\pm 0.2 \\
\end{array}$ \\
\hline & $\begin{array}{l}\text { E. segmen- } \\
\text { tinotatus }\end{array}$ & $\begin{array}{c}4.2 \pm \\
0.2 \\
\end{array}$ & $\begin{array}{c}0.7 \\
\pm 0.2 \\
\end{array}$ & $\begin{array}{l}0.1 \pm \\
0.05\end{array}$ & $\begin{array}{c}589.3 \\
\pm 4 \\
\end{array}$ & $\begin{array}{c}467.3 \\
\pm 2.5 \\
\end{array}$ & $0 \pm 0$ & $\begin{array}{r}57.3 \\
\pm 2.5 \\
\end{array}$ & $\begin{array}{l}19.2 \\
\pm 0.2 \\
\end{array}$ & $\begin{array}{r}50.6 \\
\pm 1.1 \\
\end{array}$ & $\begin{array}{c}15.3 \\
\pm 1 \\
\end{array}$ & $\begin{array}{l}18.8 \\
\pm 0.7 \\
\end{array}$ & $\begin{array}{r}41.3 \\
\pm 1.5 \\
\end{array}$ & $\begin{array}{l}2 \pm \\
0.1 \\
\end{array}$ & $\begin{array}{r}134.6 \\
\pm 2.5 \\
\end{array}$ \\
\hline \multirow[t]{3}{*}{5} & Water & $\begin{array}{c}402 \pm \\
3.2 \\
\end{array}$ & $\begin{array}{l}80.6 \\
\pm 0.5 \\
\end{array}$ & $\begin{array}{l}17.5 \\
\pm 1.3 \\
\end{array}$ & $\begin{array}{c}5.8 \\
\pm 0.2 \\
\end{array}$ & $\begin{array}{c}2.2 \\
\pm 0.2 \\
\end{array}$ & $\begin{array}{c}0.6 \pm \\
0.2 \\
\end{array}$ & $\begin{array}{c}0.4 \\
\pm 0.1 \\
\end{array}$ & $\begin{array}{c}0.13 \\
\pm 0.05 \\
\end{array}$ & $0 \pm 0$ & $\begin{array}{l}0.1 \pm \\
0.05\end{array}$ & $0 \pm 0$ & $\begin{array}{c}0.12 \\
\pm 0.03 \\
\end{array}$ & $0 \pm 0$ & $\begin{array}{l}1.9 \pm \\
0.11\end{array}$ \\
\hline & Sediment & $\begin{array}{l}0.3 \pm \\
0.05\end{array}$ & $0 \pm 0$ & $0 \pm 0$ & $0 \pm 0$ & $\begin{array}{l}657.6 \\
\pm 2.5 \\
\end{array}$ & $0 \pm 0$ & $\begin{array}{c}4.9 \\
\pm 0.6 \\
\end{array}$ & $\begin{array}{c}2.2 \\
\pm 0.2 \\
\end{array}$ & $\begin{array}{c}1 \pm \\
0.05\end{array}$ & $\begin{array}{c}1.9 \\
\pm 0.2 \\
\end{array}$ & $\begin{array}{c}2.2 \\
\pm 0.2 \\
\end{array}$ & $\begin{array}{c}1.2 \\
\pm 0.2 \\
\end{array}$ & $\begin{array}{c}1.6 \\
\pm 0.5 \\
\end{array}$ & $\begin{array}{c}0.5 \pm \\
0.2 \\
\end{array}$ \\
\hline & $\begin{array}{l}\text { E. quadri- } \\
\text { punctatus }\end{array}$ & $0 \pm 0$ & $\begin{array}{c}0.4 \\
\pm 0.09\end{array}$ & $0 \pm 0$ & $\begin{array}{c}453.6 \\
\pm 5\end{array}$ & $\begin{array}{c}904 \\
\pm 5.2\end{array}$ & $0 \pm 0$ & $\begin{array}{r}32.3 \\
\pm 2.5\end{array}$ & $\begin{array}{r}12.3 \\
\pm 2.5\end{array}$ & $\begin{array}{r}11.6 \\
\pm 1.1\end{array}$ & $\begin{array}{c}13 \pm \\
0.2\end{array}$ & $\begin{array}{l}15.5 \\
\pm 0.5\end{array}$ & $\begin{array}{l}24.1 \\
\pm 0.8\end{array}$ & $\begin{array}{c}3.8 \\
\pm 0.3\end{array}$ & $\begin{array}{c}100 \\
\pm 2\end{array}$ \\
\hline
\end{tabular}




\begin{tabular}{|c|c|c|c|c|c|c|c|c|c|c|c|c|c|c|c|}
\hline \multirow[t]{4}{*}{6} & Water & $\begin{array}{c}260 \\
\pm 3\end{array}$ & $0 \pm 0$ & $11 \pm 1$ & $\begin{array}{c}3.5 \\
\pm 0.5\end{array}$ & $\begin{array}{c}1.23 \\
\pm 0.25\end{array}$ & $\begin{array}{c}0.5 \pm \\
0.1\end{array}$ & $\begin{array}{c}0.2 \\
\pm 0.1\end{array}$ & $\begin{array}{c}0.13 \\
\pm 0.05\end{array}$ & $\begin{array}{l}0.16 \\
\pm 0.1\end{array}$ & $\begin{array}{c}0.2 \\
\pm 0.1\end{array}$ & $0 \pm 0$ & $\begin{array}{l}0.18 \\
\pm 0.1\end{array}$ & $0 \pm 0$ & $\begin{array}{l}1.26 \\
\pm 0.2\end{array}$ \\
\hline & Sediment & $\begin{array}{c}0.5 \pm \\
0.1\end{array}$ & $0 \pm 0$ & $0 \pm 0$ & $0 \pm 0$ & $\begin{array}{c}857.6 \\
\pm 2.5\end{array}$ & $\begin{array}{l}20.5 \\
\pm 0.5\end{array}$ & $\begin{array}{c}8.2 \\
\pm 0.2\end{array}$ & $\begin{array}{l}2.9 \pm \\
0.15\end{array}$ & $\begin{array}{l}2.03 \\
\pm 0.3\end{array}$ & $\begin{array}{c}3.7 \\
\pm 0.2\end{array}$ & $\begin{array}{c}3.6 \\
\pm 0.2\end{array}$ & $\begin{array}{c}2.3 \\
\pm 0.2\end{array}$ & $\begin{array}{c}4.6 \\
\pm 0.4\end{array}$ & $\begin{array}{c}1.36 \pm \\
0.15\end{array}$ \\
\hline & $\begin{array}{l}\text { E. quadri- } \\
\text { punctatus }\end{array}$ & $\begin{array}{c}7.5 \pm \\
0.4\end{array}$ & $0 \pm 0$ & $\begin{array}{c}0.2 \\
\pm 0.08\end{array}$ & $\begin{array}{c}0.1 \\
\pm 0.05\end{array}$ & $\begin{array}{c}0.14 \\
\pm 0.05\end{array}$ & $0 \pm 0$ & $\begin{array}{r}74.5 \\
\pm 1.3\end{array}$ & $0 \pm 0$ & $\begin{array}{c}142 \\
\pm 2.5\end{array}$ & $\begin{array}{c}25 \pm \\
0.7\end{array}$ & $\begin{array}{l}40.6 \\
\pm 1.1\end{array}$ & $\begin{array}{c}432.6 \\
\pm 15\end{array}$ & $\begin{array}{c}3.2 \\
\pm 0.2\end{array}$ & $\begin{array}{c}504.6 \\
\pm 5\end{array}$ \\
\hline & $\begin{array}{l}\text { E. fusci- } \\
\text { pennis }\end{array}$ & $\begin{array}{l}5.2 \pm \\
0.25\end{array}$ & $0 \pm 0$ & $\begin{array}{l}0.1 \pm \\
0.07\end{array}$ & $0 \pm 0$ & $\begin{array}{c}655 \\
\pm 5\end{array}$ & $0 \pm 0$ & $\begin{array}{c}49.3 \\
\pm 2\end{array}$ & $0 \pm 0$ & $\begin{array}{l}35.3 \\
\pm 3.5\end{array}$ & $\begin{array}{c}20.8 \\
\pm 1\end{array}$ & $25 \pm 2$ & $\begin{array}{c}59.3 \\
\pm 2\end{array}$ & $\begin{array}{c}2.2 \\
\pm 0.2\end{array}$ & $\begin{array}{c}230.3 \\
\pm 2.5\end{array}$ \\
\hline \multirow[t]{4}{*}{7} & Water & $\begin{array}{r}573 \\
\pm 3.5\end{array}$ & $0 \pm 0$ & $\begin{array}{l}25.6 \\
\pm 0.7\end{array}$ & $\begin{array}{l}6.9 \\
\pm 2\end{array}$ & $\begin{array}{c}3.9 \pm \\
0.2\end{array}$ & $\begin{array}{c}1.2 \\
\pm 0.2\end{array}$ & $\begin{array}{c}0.6 \\
\pm 0.2\end{array}$ & $\begin{array}{c}0.2 \pm \\
0.1\end{array}$ & $0 \pm 0$ & $\begin{array}{c}0.2 \\
\pm 0.1\end{array}$ & $0 \pm 0$ & $\begin{array}{c}0.27 \\
\pm 0.06\end{array}$ & $\begin{array}{c}0.23 \\
\pm 0.05\end{array}$ & $\begin{array}{l}2.9 \pm \\
0.36\end{array}$ \\
\hline & Sediment & $\begin{array}{c}0.4 \\
\pm 0.2\end{array}$ & $\begin{array}{c}0.1 \\
\pm 0.01\end{array}$ & $0 \pm 0$ & $\begin{array}{l}69.6 \\
\pm 1.5\end{array}$ & $\begin{array}{r}949 \\
\pm 6.5\end{array}$ & $0 \pm 0$ & $\begin{array}{c}5.7 \\
\pm 1.1\end{array}$ & $0 \pm 0$ & $\begin{array}{c}1.4 \\
\pm 0.2\end{array}$ & $\begin{array}{c}3.2 \\
\pm 0.2\end{array}$ & $\begin{array}{c}3.5 \\
\pm 0.5\end{array}$ & $\begin{array}{c}2.9 \\
\pm 0.55\end{array}$ & $\begin{array}{l}5.3 \pm \\
0.15\end{array}$ & $\begin{array}{c}1.03 \pm \\
0.05\end{array}$ \\
\hline & E. bicolor & $\begin{array}{r}439 \\
\pm 4.5\end{array}$ & $0 \pm 0$ & $15 \pm 3$ & $0 \pm 0$ & $\begin{array}{c}3.9 \\
\pm 0.2\end{array}$ & $\begin{array}{c}0.6 \pm \\
0.2\end{array}$ & $\begin{array}{c}0.2 \\
\pm 0.15\end{array}$ & $\begin{array}{c}0.13 \\
\pm 0.05\end{array}$ & $\begin{array}{l}0.1 \pm \\
0.05\end{array}$ & $0 \pm 0$ & $\begin{array}{l}0.1 \pm \\
0.05\end{array}$ & $\begin{array}{c}0.2 \\
\pm 0.09\end{array}$ & $0 \pm 0$ & $\begin{array}{l}3.7 \pm \\
0.25\end{array}$ \\
\hline & $\begin{array}{l}\text { E. fusci- } \\
\text { pennis }\end{array}$ & $\begin{array}{c}0.1 \\
\pm 0.05\end{array}$ & $0 \pm 0$ & $\begin{array}{c}74 \\
\pm 1.8\end{array}$ & $22 \pm 2$ & $\begin{array}{l}19.6 \\
\pm 1.5\end{array}$ & $0 \pm 0$ & $\begin{array}{c}1.9 \\
\pm 0.1\end{array}$ & $\begin{array}{c}0.6 \\
\pm 0.1\end{array}$ & $\begin{array}{c}0.4 \pm \\
0.2\end{array}$ & $\begin{array}{c}0.6 \\
\pm 0.1\end{array}$ & $\begin{array}{c}0.6 \\
\pm 0.15\end{array}$ & $\begin{array}{l}0.9 \pm \\
0.17\end{array}$ & $\begin{array}{c}0.06 \\
\pm 0.02\end{array}$ & $\begin{array}{l}8.9 \pm \\
0.25\end{array}$ \\
\hline \multirow[t]{3}{*}{8} & Water & $\begin{array}{r}203 \\
\pm 3.6\end{array}$ & $\begin{array}{c}35.6 \\
\pm 3 \\
\end{array}$ & $\begin{array}{c}0.3 \\
\pm 0.1\end{array}$ & $\begin{array}{l}2.9 \pm \\
0.17\end{array}$ & $\begin{array}{c}1.2 \\
\pm 0.25\end{array}$ & $\begin{array}{l}0.3 \pm \\
0.15\end{array}$ & $\begin{array}{c}0.1 \\
\pm 0.05\end{array}$ & $\begin{array}{l}0.1 \\
\pm 0 \\
\end{array}$ & $0 \pm 0$ & $0 \pm 0$ & $0 \pm 0$ & $\begin{array}{c}0.07 \\
\pm 0.02 \\
\end{array}$ & $0 \pm 0$ & $\begin{array}{c}0.7 \pm \\
0.1\end{array}$ \\
\hline & Sediment & $\begin{array}{l}70 \\
\pm 1 \\
\end{array}$ & $0 \pm 0$ & $\begin{array}{r}321 \\
\pm 1.5 \\
\end{array}$ & $0 \pm 0$ & $\begin{array}{c}855 \\
\pm 5 \\
\end{array}$ & $18 \pm 2$ & $\begin{array}{c}6.8 \\
\pm 1.6 \\
\end{array}$ & $\begin{array}{l}4 \pm \\
0.4\end{array}$ & $\begin{array}{c}2.1 \pm \\
0.2\end{array}$ & $\begin{array}{c}3.7 \pm \\
0.2\end{array}$ & $\begin{array}{l}4.06 \\
\pm 0.6\end{array}$ & $\begin{array}{c}2.5 \\
\pm 0.4\end{array}$ & $\begin{array}{l}7.2 \pm \\
0.25\end{array}$ & $\begin{array}{l}1.16 \\
\pm 0.2\end{array}$ \\
\hline & $\begin{array}{l}\text { E. fusci- } \\
\text { pennis }\end{array}$ & $\begin{array}{c}5.8 \\
\pm 0.3\end{array}$ & $0 \pm 0$ & $\begin{array}{c}0.1 \\
\pm 0.07\end{array}$ & $0 \pm 0$ & $\begin{array}{c}0.1 \\
\pm 0.07\end{array}$ & $0 \pm 0$ & $\begin{array}{l}54.1 \\
\pm 1.8\end{array}$ & $\begin{array}{c}26 \pm \\
1.6\end{array}$ & $\begin{array}{l}22 \\
\pm 2\end{array}$ & $\begin{array}{c}23 \pm \\
1.8\end{array}$ & $\begin{array}{l}26.3 \\
\pm 1.8\end{array}$ & $\begin{array}{l}54.5 \\
\pm 3.2\end{array}$ & $\begin{array}{l}4.16 \\
\pm 0.2\end{array}$ & $\begin{array}{c}343.3 \\
\pm 4.1\end{array}$ \\
\hline \multirow[t]{3}{*}{9} & Water & $\begin{array}{r}390 \\
\pm 5.5\end{array}$ & $0 \pm 0$ & $\begin{array}{l}16.2 \\
\pm 1.5 \\
\end{array}$ & $\begin{array}{c}5.5 \\
\pm 0.5 \\
\end{array}$ & $\begin{array}{c}2.9 \\
\pm 0.15 \\
\end{array}$ & $\begin{array}{c}0.7 \pm \\
0.2\end{array}$ & $\begin{array}{c}0.2 \\
\pm 0.15\end{array}$ & $\begin{array}{c}0.16 \pm \\
0.05\end{array}$ & $\begin{array}{l}0.1 \\
\pm 0\end{array}$ & $\begin{array}{l}0.2 \pm \\
0.05\end{array}$ & $0 \pm 0$ & $\begin{array}{c}0.16 \\
\pm 0.05\end{array}$ & $0 \pm 0$ & $\begin{array}{c}1.8 \\
\pm 0.3\end{array}$ \\
\hline & Sediment & $\begin{array}{c}0.5 \\
\pm 0.2\end{array}$ & $0 \pm 0$ & $\begin{array}{c}290 \\
\pm 3\end{array}$ & $0 \pm 0$ & $\begin{array}{c}927.3 \\
\pm 2.5\end{array}$ & $17 \pm 2$ & $\begin{array}{c}7.5 \\
\pm 0.1\end{array}$ & $0 \pm 0$ & $\begin{array}{c}1.6 \\
\pm 0.5\end{array}$ & $\begin{array}{c}3.7 \pm \\
0.2\end{array}$ & $\begin{array}{c}2.8 \\
\pm 0.7\end{array}$ & $\begin{array}{c}2.5 \\
\pm 0.3\end{array}$ & $\begin{array}{c}3.5 \\
\pm 0.5\end{array}$ & $\begin{array}{l}0.9 \pm \\
0.17\end{array}$ \\
\hline & $\begin{array}{l}\text { E. quadri- } \\
\text { punctatus }\end{array}$ & $\begin{array}{l}47 \\
\pm 2\end{array}$ & $\begin{array}{c}8.3 \\
\pm 0.2\end{array}$ & $\begin{array}{c}5.7 \\
\pm 0.2\end{array}$ & $\begin{array}{c}1.7 \\
\pm 0.3\end{array}$ & $\begin{array}{c}0.7 \\
\pm 0.12\end{array}$ & $\begin{array}{c}0.5 \pm \\
0.2\end{array}$ & $\begin{array}{c}0.1 \\
\pm 0.04\end{array}$ & $\begin{array}{c}952 \\
\pm 6.2\end{array}$ & $\begin{array}{c}531.3 \\
\pm 7\end{array}$ & $0 \pm 0$ & $\begin{array}{c}100.3 \\
\pm 2.5\end{array}$ & $\begin{array}{l}62.3 \\
\pm 2.5\end{array}$ & $\begin{array}{c}72.3 \pm \\
2.5\end{array}$ & $\begin{array}{c}166.3 \\
\pm 3.2\end{array}$ \\
\hline
\end{tabular}

Table 3: Heavy element concentration of (Mean \pm SD) in water, sediment and Enochrus spp. (ppm)

\section{Results}

In total, 191 individuals of Hydrophilidae (Coleoptera), belonging to genus Enochrus spp. were collected from 9 stations. Six species of genus Enochrus Thomson 1859 were determined. Determined species are as follows; Enochrus (Lumetus) ater (Kuwert 1888), Enochrus (L.) segmentinotatus (Kuwert 1888), Enochrus (L.) bicolor (Fabricius 1792), Enochrus (L.) quadripunctatus (Herbst 1797), Enochrus (L.) fuscipennis (Thomson 1884), Enochrus (L.) halophilus (Bedel 1878).

In general, values of some elements like $\mathrm{Ti}, \mathrm{Cr}$ and $\mathrm{Pb}$ are higher in almost all the samples. In all stations of water and sediment samples $\mathrm{Ti}, \mathrm{Fe}, \mathrm{Ni}, \mathrm{Br}$, and $\mathrm{Pb}$ were measured. In all Enochrus species $\mathrm{Fe}, \mathrm{Ni}, \mathrm{Se}$, and $\mathrm{Pb}$ were measured. The most abundant elements in Enochrus spp. were $\mathrm{Ti}, \mathrm{Cr}, \mathrm{Mn}, \mathrm{Fe}, \mathrm{Ni}, \mathrm{Cu}, \mathrm{Zn}, \mathrm{As}, \mathrm{Se}, \mathrm{Br}$, and $\mathrm{Pb}$.

Sediment concentration results show that, in all stations $\mathrm{Ti}$, Fe, $\mathrm{Ni}, \mathrm{Zn}, \mathrm{As}, \mathrm{Se}, \mathrm{Br}, \mathrm{Sr}$, and $\mathrm{Pb}$ were measured. Besides, the highest concentration of $\mathrm{Cr}$ was measured in stations 1,2,3,4, and 9; the highest concentration of Fe was measured in stations 5, 6, 7, 8 and 9; the highest concentration of Ti was in stations 8; the highest concentration of Mn was in stations 3. V measured only stations 1 and 7. Heavy element levels in water indicated that $\mathrm{Ti}$, and $\mathrm{V}$ had the highest concentration in all stations. Only station 7 had Sr concentration, but there is no Se and Sr concentration in water of all rest stations. In water, the rest of the heavy elements were in lowest concentration. The element concentration in water samples are arranged as $\mathrm{Ti}>\mathrm{V}>\mathrm{Cr}>\mathrm{Mn}>\mathrm{Fe}>\mathrm{Pb}>\mathrm{Co}>\mathrm{Ni}$ $>\mathrm{Cu}>\mathrm{As}>\mathrm{Zn}>\mathrm{Br}>\mathrm{Sr}>\mathrm{Se}$ and in sediment samples $\mathrm{Cr}>\mathrm{Fe}>\mathrm{Mn}>\mathrm{Ni}>\mathrm{Co}>\mathrm{Se}>\mathrm{Sr}>\mathrm{As}>\mathrm{Cu}>\mathrm{Br}>\mathrm{Zn}>\mathrm{Pb}>\mathrm{Ti}>\mathrm{V}$. Results of this study indicates that E. quadripunctatus is the best accumulator for certain elements, and followed by E. fuscipennis and $E$. segmentinotatus. Compared to the other Enochrus species, it appears that E. quadripunctatus accumulated the highest level of Ti, $\mathrm{Fe}, \mathrm{Ni}, \mathrm{Cu}, \mathrm{Zn}, \mathrm{As}, \mathrm{Se}, \mathrm{Br}, \mathrm{Sr}$, and $\mathrm{Pb}$. The highest concentrations of rest of the elements; in E. bicolor was $\mathrm{V}$, in E. halophilus $\mathrm{Co}$, in E. ater $\mathrm{Cr}$, in E. segmentinotatus Mn.

The water quality levels were compared according to Turkish Water Pollution and Control Regulations (TWPCR 2004) [21]. There are four quality classes according to TWPCR: high quality water (I), weakly polluted water (II), polluted water (III), and highly polluted water (IV). According to TWPCR, water in all stations have highly polluted (IV) and polluted (III) water quality in terms of $\mathrm{Pb}, \mathrm{As}, \mathrm{Cr}, \mathrm{Co}, \mathrm{Ni}, \mathrm{Mn}$, and Se; weakly polluted water quality (II) in regard to Fe, third and fourth stations except to this; high quality water (I) in regard to $\mathrm{Zn}$ and Ba. Only the fourth station has Se concentration and has IV water quality. According to the results it was determined that these concentrations adversely affected water quality. The stations 1, 2, 3, 5, 7, and 9 have III and IV water quality in regard to $\mathrm{Pb}, \mathrm{As}, \mathrm{Cr}, \mathrm{Co}, \mathrm{Ni}$, and $\mathrm{Mn}$; this can be due to cement factory spreading ash emission, traffic pollution due to highways, and agriculture activities in Gökçeyamaç village. Station 4, 6, and 8 has II water quality in regard to Fe and rests 
of the stations have IV and III water quality degree. These results are given in Table 4. Determination of $\mathrm{Pb}, \mathrm{As}, \mathrm{Cr}, \mathrm{Co}, \mathrm{Ni}$, and Mn residues in water samples at the stations 7, and 8 where human activity is limited, reveals the extent of the spread. Because in these stations, there are no intensive agriculture or traffic. Ce, Pd, Ba, In, Sn, Nb, Pm, La, and Mo were below the detection limit of EDXRF spectrometry (ppm).

\begin{tabular}{|c|c|c|c|c|c|c|c|c|c|c|c|c|c|}
\hline \multirow{2}{*}{$\begin{array}{l}\text { Heavy } \\
\text { Elements }\end{array}$} & \multirow{2}{*}{$\begin{array}{c}\text { Station } \\
1\end{array}$} & \multirow{2}{*}{$\begin{array}{c}\text { Station } \\
2\end{array}$} & \multirow{2}{*}{$\begin{array}{c}\text { Station } \\
3\end{array}$} & \multirow{2}{*}{$\begin{array}{c}\text { Station } \\
4\end{array}$} & \multirow{2}{*}{$\begin{array}{c}\text { Station } \\
5\end{array}$} & \multirow{2}{*}{$\begin{array}{c}\text { Station } \\
6\end{array}$} & \multirow{2}{*}{$\begin{array}{c}\text { Station } \\
7\end{array}$} & \multirow{2}{*}{$\begin{array}{c}\text { Station } \\
8\end{array}$} & \multirow{2}{*}{$\begin{array}{c}\text { Station } \\
9\end{array}$} & \multicolumn{4}{|c|}{ TWPCR $(\mu \mathrm{g} / \mathrm{L})$} \\
\hline & & & & & & & & & & I & II & III & IV \\
\hline $\mathrm{Pb}$ & $\begin{array}{l}1.7 \pm \\
0.25\end{array}$ & $\begin{array}{c}1.9 \pm \\
0.2\end{array}$ & $\begin{array}{l}3.23 \\
\pm 0.2\end{array}$ & $\begin{array}{l}0.9 \pm \\
0.05\end{array}$ & $\begin{array}{l}1.9 \pm \\
0.11\end{array}$ & $\begin{array}{c}1.26 \pm \\
0.2\end{array}$ & $\begin{array}{l}2.9 \pm \\
0.36\end{array}$ & $\begin{array}{c}0.7 \pm \\
0.1\end{array}$ & $\begin{array}{c}1.8 \\
\pm 0.3\end{array}$ & 10 & 20 & 50 & $>50$ \\
\hline As & $\begin{array}{c}0.2 \\
\pm 0.1\end{array}$ & $\begin{array}{c}0.2 \\
\pm 0.1\end{array}$ & $\begin{array}{c}0.2 \\
\pm 0.1\end{array}$ & $\begin{array}{c}0.1 \pm \\
0.5\end{array}$ & $\begin{array}{l}0.1 \pm \\
0.05\end{array}$ & $\begin{array}{c}0.2 \pm \\
0.1\end{array}$ & $\begin{array}{c}0.2 \pm \\
0.1\end{array}$ & $0 \pm 0$ & $\begin{array}{l}0.2 \pm \\
0.05\end{array}$ & 20 & 50 & 100 & $>100$ \\
\hline $\mathrm{Cu}$ & $\begin{array}{c}0.36 \\
\pm 0.15\end{array}$ & $\begin{array}{c}0.13 \\
\pm 0.05\end{array}$ & $\begin{array}{c}0.2 \\
\pm 0.1\end{array}$ & $\begin{array}{l}0.1 \pm \\
0.05\end{array}$ & $\begin{array}{c}0.13 \pm \\
0.05\end{array}$ & $\begin{array}{c}0.13 \pm \\
0.05\end{array}$ & $\begin{array}{c}0.2 \pm \\
0.1\end{array}$ & $\begin{array}{l}0.1 \\
\pm 0\end{array}$ & $\begin{array}{c}0.16 \pm \\
0.05\end{array}$ & 20 & 50 & 200 & $>200$ \\
\hline $\mathrm{Cr}$ & $\begin{array}{c}22 \pm \\
2.6\end{array}$ & $\begin{array}{r}14.6 \\
\pm 2.5\end{array}$ & $\begin{array}{l}10.3 \\
\pm 0.2\end{array}$ & $\begin{array}{c}10.2 \pm \\
0.6\end{array}$ & $\begin{array}{r}17.5 \\
\pm 1.3\end{array}$ & $11 \pm 1$ & $\begin{array}{r}25.6 \\
\pm 0.7\end{array}$ & $\begin{array}{c}0.3 \pm \\
0.1\end{array}$ & $\begin{array}{c}16.2 \pm \\
1.5\end{array}$ & 20 & 50 & 200 & $>200$ \\
\hline Co & $\begin{array}{l}1 \pm \\
0.1\end{array}$ & $\begin{array}{c}0.03 \\
\pm 0.02\end{array}$ & $\begin{array}{c}1.8 \\
\pm 0.2\end{array}$ & $\begin{array}{c}0.3 \pm \\
0.1\end{array}$ & $\begin{array}{c}0.6 \\
\pm 0.2\end{array}$ & $\begin{array}{c}0.5 \pm \\
0.1\end{array}$ & $\begin{array}{c}1.2 \pm \\
0.2\end{array}$ & $\begin{array}{l}0.3 \pm \\
0.15\end{array}$ & $\begin{array}{c}0.7 \pm \\
0.2\end{array}$ & 10 & 20 & 200 & $>200$ \\
\hline $\mathrm{Ni}$ & $\begin{array}{c}0.3 \pm \\
0.2\end{array}$ & $\begin{array}{c}0.5 \\
\pm 0.1\end{array}$ & $\begin{array}{c}0.7 \pm \\
0.2\end{array}$ & $\begin{array}{c}0.2 \pm \\
0.1\end{array}$ & $\begin{array}{c}0.4 \\
\pm 0.1\end{array}$ & $\begin{array}{c}0.2 \pm \\
0.1\end{array}$ & $\begin{array}{c}0.6 \pm \\
0.2\end{array}$ & $\begin{array}{c}0.16 \pm \\
0.05\end{array}$ & $\begin{array}{c}0.23 \pm \\
0.15\end{array}$ & 20 & 50 & 200 & $>200$ \\
\hline $\mathrm{Zn}$ & $0 \pm 0$ & $\begin{array}{c}0.2 \\
\pm 0.1\end{array}$ & $\begin{array}{c}0.2 \\
\pm 0.1\end{array}$ & $\begin{array}{l}0.1 \\
\pm 0\end{array}$ & $0 \pm 0$ & $\begin{array}{c}0.16 \pm \\
0.1\end{array}$ & $0 \pm 0$ & $0 \pm 0$ & $0.1 \pm 0$ & 200 & 500 & 2000 & $>2000$ \\
\hline $\mathrm{Fe}$ & $\begin{array}{l}2.9 \pm \\
0.15\end{array}$ & $\begin{array}{c}2.2 \\
\pm 0.2\end{array}$ & $\begin{array}{c}4.8 \\
\pm 0.4\end{array}$ & $\begin{array}{c}2.1 \\
\pm 0.4\end{array}$ & $\begin{array}{c}2.2 \pm \\
0.2\end{array}$ & $\begin{array}{c}1.23 \pm \\
0.25\end{array}$ & $3.9 \pm 0.2$ & $\begin{array}{l}1.2 \pm \\
0.25\end{array}$ & $\begin{array}{l}2.9 \pm \\
0.15\end{array}$ & 300 & 1000 & 5000 & $>5000$ \\
\hline $\mathrm{Mn}$ & $\begin{array}{l}6.2 \pm \\
0.26\end{array}$ & $\begin{array}{c}4.9 \\
\pm 0.6\end{array}$ & $\begin{array}{l}10.1 \\
\pm 0.7\end{array}$ & $\begin{array}{c}3.3 \\
\pm 0.2\end{array}$ & $\begin{array}{c}5.8 \\
\pm 0.2\end{array}$ & $\begin{array}{c}3.5 \pm \\
0.5\end{array}$ & $6.9 \pm 2$ & $\begin{array}{l}2.9 \pm \\
0.17\end{array}$ & $\begin{array}{c}5.5 \pm \\
0.5\end{array}$ & 100 & 500 & 3000 & $>3000$ \\
\hline $\mathrm{Se}$ & $0 \pm 0$ & $0 \pm 0$ & $0 \pm 0$ & $\begin{array}{c}0.1 \\
\pm 0.1\end{array}$ & $0 \pm 0$ & $0 \pm 0$ & $0 \pm 0$ & $0 \pm 0$ & $0 \pm 0$ & 10 & 10 & 20 & $>20$ \\
\hline $\mathrm{Ba}$ & $\begin{array}{c}0,00 \\
71\end{array}$ & $\begin{array}{c}0,00 \\
53\end{array}$ & $\begin{array}{c}0,01 \\
14\end{array}$ & $\begin{array}{c}0,0 \\
039\end{array}$ & $\begin{array}{c}0,00 \\
62\end{array}$ & $\begin{array}{c}0,0 \\
004\end{array}$ & $\begin{array}{c}0,0 \\
093\end{array}$ & $\begin{array}{c}0,0 \\
031\end{array}$ & $\begin{array}{c}0,0 \\
063\end{array}$ & 1000 & 2000 & 2000 & $>2000$ \\
\hline
\end{tabular}

Table 4: The results for water were compared with national water regulation (TWPCR) (ppm)

\section{Discussion}

Aquatic insects are inseparable part of freshwater ecosystems. When food chain is considered, pollutions are able to reach to another organism from one to another, and also at the end human beings. Therefore, protecting the wetlands in turn can protect our safety and welfare. Thus, this research attempted to determine the potential use of aquatic insect communities as biomonitor as well as assess the health status of wetlands in Erzurum province and surrounding.

The concentrations of the fourteen elements were measured different level in insects, water and sediment. The highest pollution levels were found in the samples taken from the industrial sites $(1,2,4,5)$. In field study it was observed that insect samples have abundance in industrial sites and same results were obtained by Pakulnicka et al. (2015) [22]. The element concentrations were found to increase from water to insects in general (water<sediment<Enochrus spp.). Thus, it can be said that, heavy elements would be transported to organisms on the higher position from water to insects and along food chains. The studies of Boyd (2009) [23] and Aydoğan et al. (2017) [8] support that result.

In the present study it can be seen that, contaminants residues in Enochrus spp. are reflective of environmental quality. The measured element concentrations reflect a clear influence of anthropogenic activities. Element concentration in the Enochrus spp. showed differences in accordance to the sediment contamination of each station. High concentrations of the elements in sediment and water samples cause high level of heavy elements in Enochrus spp. The heavy element concentrations varied significantly by the sampling sites and species. Station 2, 3, and 4, where anthropogenic activities are intense, have the highest number of individuals. Enochrus spp. can survive even the locations which they live in low environmental quality. Therefore, it can be thought that they are tolerant to metal pollution. The results also show that it is possible to analyze element concentrations in these aquatic insects. Enochrus spp. fulfill the aforementioned biomonitor criteria like represented in large numbers all over the monitoring area, have numerical abundance, cosmopolitan distribution and easy identifiable.

It is known that proportion to the volume of traffic and close distance from the road lead contribution of Pb level environment and carried by aerial deposition alongside the road [24]. Besides to traffic pollution, industrial activity is major source of heavy metals/metalloids [3]. But some of the stations are away from anthropogenic sources and this can be explained by only atmospheric transportation [25]. 
To summarize, the data given in this study showed that Enochrus spp. are capable of reflecting element bioaccumulation with different levels and embodies all these criteria, thus Enochrus spp. can be used in environmental quality monitoring studies. For more information to assess health of environment, long-term biomonitoring must be regularly done. Because long-term biomonitoring of aquatic insect communities may provide insight into how human induced alterations are affecting community structure, ecosystem functions and water quality. It is hoped that the results from this study will provide baseline information in Erzurum province.

\section{Acknowledgement}

This research, has been supported by project of the Atatürk University Scientific Research Project SRP-2012/162, and is a part of first author's Ph. D thesis. The authors express their sincere gratitude Assist. Prof. Dr. Buğrahan EMSEN of the department of Biology at the University Of Karamanoğlu Mehmet Bey, for statistical analysis of samples.

\section{References}

1. Ololade IA, Lajide L, Amoo IA, Oladoja NA (2008) Investigation of heavy metals contamination of edible marine seafood. Afr J Pure Appl Chem 2: 121-31.

2. Rainbow PS (2002) Trace metal concentrations in aquatic invertebrates: why and so what? Environ Pollut 120: 497-507.

3. Depledge MH, Weeks JM, Bjerregaard P (1994) Heavy metals. In: Handbook of ecotoxicology, Blackwell Scientific, London: UK.

4. Breure AM, Markert BA, Zechmeister HG (2003) Bioindicators \& Biomonitors: Principles, Concepts and Applications. Elsevier.

5. Wittig R (1993) General aspects of biomonitoring heavy metals by plants. In: Markert B (ed) Plants as biomonitors: indicators for heavy metals in the terrestrial environment. Weinheim VCH Publisher, 3-27.

6. Markert BA, Wappelhorst O, Weckert V, Herpin U, Siewers U, et al. (1999) The use of bioindicators for monitoring the heavy metal status of the environment. J Radioanal and Nucl Ch 240: 425-9.

7. Benetti CJ, Pérez-Bilbao A, Garrido J (2012) Macroinvertebrates as indicators of water quality in running waters: 10 years of research in rivers with different degrees of anthropogenic impacts. In Ecological Water Quality-Water Treatment and Reuse. InTech.

8. Aydoğan Z, Şişman T, İncekara Ü, Gürol A (2017) Heavy metal accumulation in some aquatic insects (Coleoptera: Hydrophilidae) and tissues of Chondrostoma regium (Heckel, 1843) relevant to their concentration in water and sediments from Karasu River, Erzurum, Turkey. Environ Sci Pollut Res Int 24: $9566-9574$.

9. Tardiff RG, Goldstein BD (1991) Methods for assessing exposure of human and non-human biota.

10. Pennak RW (1978) Freshwater invertebrates of the United States, 2nd ed. John Wiley and Sons, New York, 810.

11. Arimoro FO, Ikomi RB (2009) Ecological Integrity of upper Warri River, Niger Delta using aquatic insects as bioindicators. Ecological Indicators 9: 455-61.

12. Merritt RW, Cummins KW (1996) An introduction to the aquatic insects of North America. 3rd ed. Kendall-Hunt, Dubuque, IOWA, 862.

13. Eugenia López-López, Jacinto Elías Sedeño-Díaz (2015) Biological indicators of water quality: The role of fish and macroinvertebrates as indicators of water quality. Environmental Indicators. Springer, Dordrecht

14. Nasirian H, Irvine KN (2017) Odonata larvae as a bioindicator of metal contamination in aquatic environments: application to ecologically important wetlands in Iran. Environmental monitoring and assessment, 189: 436.

15. Fikáček M, Gentili E, Short AEZ (2010) Order Coleoptera, family Hydrophilidae. Arthropod fauna of the UAE 3: 135-65.

16. Tara JS, Kour R, Sharma S (2011) Studies on the occurrence and seasonal abundance of aquatic Coleoptera in relation to some physicochemical parameters of water of Gharana wetland reserve Jammu (J and K). The Bioscan 6: 257-61.

17. Lee DH and Ahn KJ (2016) A taxonomic review of Enochrus Thomson (Coleoptera: Hydrophilidae) in Korea. J Asia-Pacific Biodiversity 9: 335-43.

18. Aydoğan Z, Gürol A, İncekara Ü (2016) The investigation of heavy element accumulation in some Hydrophilidae (Coleoptera) species. Environmental monitoring and assessment 188: 204.

19. Hansen M (1987) The Hydrophilidae (Coleoptera) of Fennoscandia and Denmark, 18. Fauna Entomologica Scandinavica, EJ Brill, Denmark, 253.

20. Hansen M (1991) The Hydrophiloid beetles phylogeny, classification and a revision of the genera (Coleoptera, Hydrophiloidea). Biol Skr, 40, 365.

21. TWPCR (2004) Turkish water pollution control regulations-25684 (in Turkish). Ministry of environment and urbanization, 25684 oficial gazette, Ankara.

22. Pakulnicka J, Buczyńska E, Buczyński P, Czachorowski S, Kurzątkowska A, et al. (2015) Are beetles good indicators of insect diversity in freshwater lakes?. Oceanological and Hydrobiological Studies 44: 487-99.

23. Boyd RS (2009) High-nickel insects and nickel hyperaccumulator plants: A review. Insect Science 16: 19-31. Doi 10.1111/j.1744-7917.2009.00250.x.

24. Raj SP and Ram PA (2013) Determination and contamination assessment of Pb, Cd, and Hg in roadside dust along Kathmandu-Bhaktapur road section of Arniko Highway, Nepal. Res J Chem Sci 3: 18-25

25. Chen WK, Li TC, Sheu GR, Lin NH, Chen LY, et al. (2016) Correlation analysis, transportation mode of atmospheric mercury and criteria air pollutants, with meteorological parameters at two remote sites of mountain and offshore island in Asia. Aerosol Air Qual Res 16: 2692-705. 


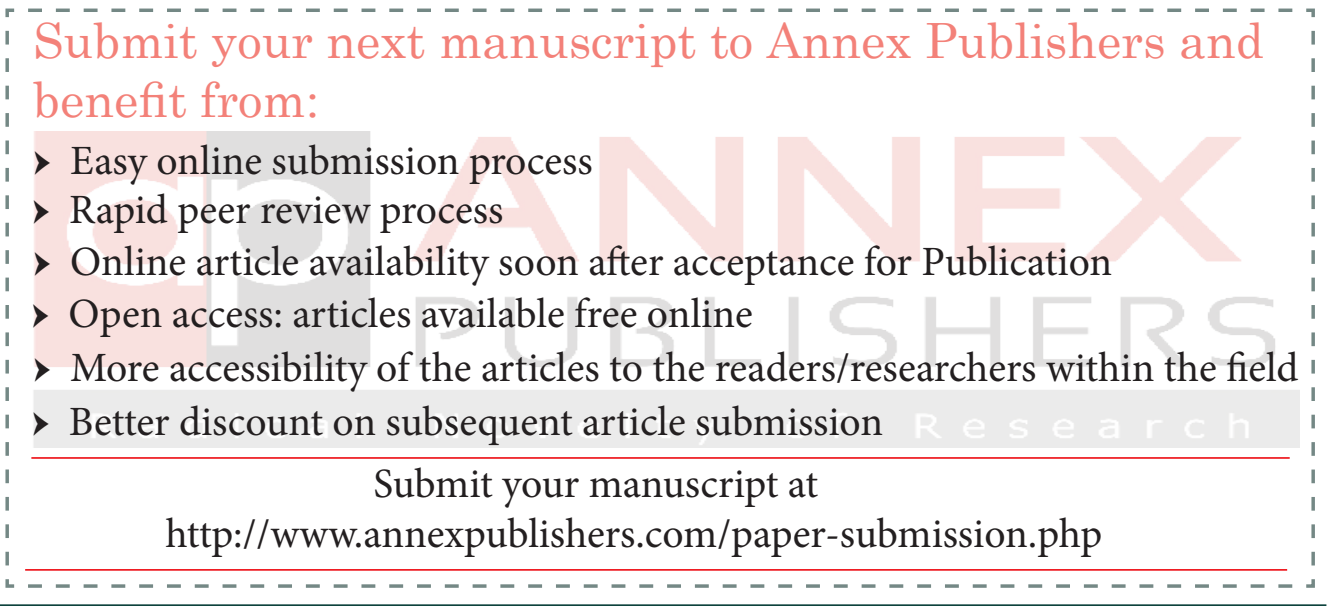

